\title{
ICONICITY AND APE GESTURE
}

\author{
MARCUS PERLMAN \\ Cognitive and Information Sciences, University of California, Merced \\ 5200 N Lake Rd, Merced, CA 95343, USA \\ NATHANIEL CLARK \\ Cognitive Psychology, University of California, Santa Cruz \\ 1156 High St, Santa Cruz, CA 95064, USA
}

JOANNE E. TANNER

Centre for Social Learning and Cognitive Evolution, Scottish Primate Research Group School of Psychology, University of St. Andrews Home address: 3071 Dover Drive, Santa Cruz, CA 95065, USA

Iconic gestures are hypothesized to be crucial to the evolution of language. Yet the important question of whether apes produce iconic gestures is the subject of considerable debate. This paper presents the current state of research on iconicity in ape gesture. In particular, it describes some of the empirical evidence suggesting that apes produce three different kinds of iconic gestures; it compares the iconicity hypothesis to other major hypotheses of ape gesture; and finally, it offers some directions for future ape gesture research.

\section{Introduction}

Several major theories point to the human ability to create iconic gestures as fundamental to the evolution of language (e.g., Arbib, 2012; Armstrong \& Wilcox, 2007; Tomasello, 2008). Thus the question of whether apes can create iconic gestures has great significance for theories of how human language and gesticulation evolved from the communication of our common ancestor. Yet the answer to this question is the subject of considerable disagreement in the field of ape gesture research. The aim of this paper is to briefly present the current state of research on iconicity in ape gesture. We begin by describing some of the empirical evidence suggesting that apes produce, in particular, three different kinds of iconic gestures. Next we consider iconicity with respect to the main alternative theories of ape gesture. And finally, we conclude with some related considerations for future ape gesture research. 


\section{Iconicity in Ape Gesture}

The notion of what constitutes an iconic gesture differs across researchers and fields, and the term iconic often overlaps with other terms like representative, pantomimic, and mimetic. Here we use iconic generally to refer to the quality exhibited when a gesture in some way resembles or depicts its meaning, particularly when it is formed spontaneously (as opposed to being ritualized or conventionalized) ${ }^{1}$. For example, an ape might sweep its hand downward through space as an iconic gesture to depict the direction of movement desired by a partner (e.g. Savage-Rumbaugh et al., 1977), or an enculturated ape who likes to play with clay might pantomime rolling a ball of clay between her hands to request a handful of clay (Tanner et al., 2006).

Previous research has described three basic kinds of iconic gestures that are produced by apes, which we label directive touches, visible directives, and pantomimed actions. These classes may be organized along a continuum of complexity and abstraction from action, but are not necessarily distinct cognitive categories. Nevertheless, there are some qualitative differences between these types of gestures that bear significance for theories of language evolution (cf. Arbib, 2012). Notable variables relate to transitivity of the gesture (e.g. transitive, intransitive, and reflexive) and the representation of arguments (e.g. patients, themes, and instruments; Perlman \& Gibbs, accepted).

Below we describe these three kinds of gestures, present examples of each, and highlight some of the empirical studies in their support. We acknowledge at the outset that mechanistic explanations for the examples we present - for example, whether the gestures originate from iconic processes or from ritualization - is a matter of ongoing debate. Our goal, in the limited space available, is to bring attention to important issues within this debate, rather than to resolve them.

\subsection{Directive Touches}

Directive touches refer to the tactile (transitive) gestures used by one animal to direct another's movement and positioning by pushing, pulling, nudging, tugging, lifting, etc., typically with mechanically ineffective force (but see Perlman et al., 2012 regarding force). The iconicity of these gestures relates to the direction and implied force of the gesture, which communicate the particular

\footnotetext{
${ }^{1}$ Note that researchers of signed languages use the term iconic to refer to conventionalized forms too. It is debated whether the iconicity in these forms is an active part of online processing.
} 
movement that is desired of the partner. For example, a male bonobo gently touches a female's shoulder and pushes her upper torso away from himself to communicate that he would like her to turn around (Savage-Rumbaugh et al., 1977).

One relevant study describes a set of 54 directive pushes used by a captive mother gorilla to guide her infant around their enclosure (Perlman et al., 2012). These pushes were variable in form and force in ways that were tuned to the present physical and social context, indicating the underlying partial activation of afforded instrumental actions (i.e. physically effective pushes). Other directive touches are reported by Tanner and Byrne (1996), who describe such gestures used during play between a captive silverback and young female gorilla.

\subsection{Visible Directives}

Visible directives refer to gestures used by an animal to influence the movement or positioning of another by showing an aspect of the desired movement visibly, either in the air (intransitive) or on its own body (reflexive), but without directly contacting the communicative partner. For example, a chimpanzee makes reaching and beckoning gestures to request a partner to approach (e.g. Crawford, 1937; Köhler, 1925).

Tanner and Byrne (1996) observed a number of visible directive movements performed by the silverback in the previously mentioned pair of captive gorillas. His gestures illustrated the actions that he desired of the female by demonstrating a path of motion, either in space or on his own body. For example, he would swing his arm down between his legs which served to direct and encourage physical contact between them. Similar gestures were used by the group of bonobos described by Savage-Rumbaugh et al. (1977) in their negotiation of sexual positions.

\subsection{Pantomimed Actions}

Pantomimed actions are instances when an animal communicates by demonstrating a particular action, usually to get a partner to perform that action or to request an associated object. These gestures are most frequently documented in apes with extensive experience interacting with humans, and typically relate to relatively complex action routines that the ape has learned from this experience. The action routines are often object directed and can involve multiple arguments. Sometimes the pantomime incorporates the actual physical objects involved in the routine, which may scaffold the production of 
the gesture. For example, an enculturated gorilla makes a drinking gesture bringing an empty cup to her lips and tilting it back - to express that she would like a drink (Perlman \& Gibbs, accepted). Performing a similar pantomime without the cup may be a more cognitively challenging gesture to perform.

In one case, pantomimic gestures were produced by free-ranging rehabilitant orangutans (Russon and Andrews, 2011). The study identified 18 pantomimes from 20 years of written field observations. The gestures, mostly performed with human partners rather than orangutans, included directed scratch gestures with a stick, feigning inability to open fruit as a request for another to do it, and reenacting a caregiver doctoring her cut foot. There also several anecdotal reports of pantomimed actions produced by human-reared apes, such as Hayes and Nissen's (1971: 107) description of the juvenile chimpanzee Viki's gestures:

Watching bread being kneaded, she begged for a sample of dough by going through the kneading motions for a while, and then holding out her hand, palm up, moving her fingers in the gesture which means "give me" to both her species and ours. A similar incident occurred during the weekly ironing as she grew impatient for her turn to do the napkins. She stood on a nearby table, moving one clenched fist slowly back and forth above the ironing board while her other hand tried to take the iron away from 'mamma.'

(Also see Savage-Rumbaugh, et al., 1986 on human-reared bonobos and Tanner et al., 2006 on a human-reared gorilla.)

Outside of human influence, free ranging chimpanzees were observed to perform reflexive directed scratches to show partners where they would like to be scratched (Pika \& Mitani, 2006).

\section{Alternative Theories of Ape Gestures}

Much current research tends to dismiss iconicity as a potential factor in ape gesture (e.g., Arbib et al., 2008; Call \& Tomasello, 2007; Hobaiter \& Byrne, 2011). Instead, studies are primarily motivated by two major competing theories: the Ontogenetic Ritualization (OR) hypothesis of Tomasello, Call and colleagues, and the Biological Inheritance (BI) hypothesis of Byrne, Hobaiter and colleagues. Both of these theories maintain, more or less explicitly, that any apparent iconicity in an ape gesture is an inactive artifact of its ontogenetic or 
phylogenetic development. Thus it is argued that ape gestures lack the spontaneous simulative quality of human iconic gesture.

According to the process of OR, gestures are created through the iteration of recurring dyadic interactions that begin with an instrumental action. For example, an infant reaches out, grabs and tugs on his mother in order to move her toward him. As this interaction is repeated, the accommodating mother begins to anticipate her infant's goal, and becomes quicker to move towards him as she recognizes that he is reaching for her. In turn, the infant anticipates his mother's accelerated response and progressively abbreviates the reaching act until eventually a stable reach gesture is formed. Although OR is likely to be an important process in the development of some ape gestures, recent studies have failed to find the idiosyncrasy and one-way usage in gesture repertoires that is predicted by the process (Genty et al., 2009; Hobaiter \& Byrne, 2011). Another shortcoming of OR is that, while it explains how instrumental actions become abbreviated into stable gestures, it does not account for the variability of form and force that is often characteristic of ape gesturing (Perlman et al., 2012).

As an alternative to OR, Byrne and colleagues have proposed biological inheritance as the default null hypothesis because it is "the normal case for the communicative signals of most species of animal" (Hobaiter \& Byrne, 2011: 748). BI proposes that through a process of genetic channeling, ape species have evolved large species-typical gesture repertoires, a portion of which appear common to the ape family (e.g. 102 distinct gesture types in gorillas, 66 in chimpanzees). However, the theory does not explain how evolutionary processes could so quickly give rise to so many innately specified gestural forms, many of which are quite similar to each other (e.g. the posited gorilla repertoire includes 2-handed grab, 2-handed grab-pull, grab-pull, one-handed grab, positioning, hand on, hands on, 1-handed push, 2-handed push, and touch). A more parsimonious explanation may be that some of these various directive gestures are created on the fly and reflect instrumental positioning actions that are afforded by the particular social interaction, such as the relative positioning between the two animals (Perlman et al. 2012). It is also clear that BI cannot account for many of the pantomimes produced by human-reared apes.

Compared to OR and BI, a focus on iconicity leads to a more conceptually oriented approach to understanding how apes create gestures. This approach may be more compatible with certain cognitive theories of human gesture production (e.g. Hostetter \& Alibali, 2008; McNeill, 1992). For example, one hypothesis proposes that apes produce iconic gestures through a process similar to the human process of simulating actions (Perlman \& Gibbs, accepted; Perlman et al., 2012). According to this account, when apes communicate with 
each other, they sometimes produce gestures by enacting partial or mechanically ineffective versions of particular, contextually relevant actions (e.g. directive touches) or by enacting more abstracted simulations of relevant actions (e.g. visible directives and pantomimes). In turn, the communication partner understands the meaning of the gesture as it primes recognition of the relevant instrumental action.

\section{Directions for Future Research}

In general, future research and modeling of ape gesture should aim to distinguish between the primary hypotheses for the processes of ape gesture acquisition and production: ontogenetic ritualization, biological inheritance, and iconicity ${ }^{2}$. These processes do not need to be exclusive. They may all interact at different time scales to influence the dynamic forms of movement that are created during real-time gesture production. More specific issues relating to variability and enculturation are described below.

\subsection{Variability}

Ontogenetic ritualization and biological inheritance may explain stability in gestural forms, but cannot account for the considerable variability in their production (Perlman et al., 2012). This point is important because iconic gestures in human production are characteristically variable, distinguished by their analog, idiosyncratic quality (Cook \& Tanenhaus, 2008; McNeill, 1992). Human speech too, even in its high conventionality, still shows freedom for variation through prosody, which is believed to be closely related to gesture (Bolinger, 1986). Hypotheses based on iconicity may be useful to explain variability in ape gestures and thus to relate ape gesturing to human communication. Future research should examine how similar gestures vary from instance to instance, and give more rigorous attention to the source of that variation. Critically, variability poses challenges with respect to the notorious "lumping/splitting" conundrum and assessments of gestural repertoires.

\subsection{Enculturation}

How does experience interacting with humans and human culture influence ape gesturing? Evidence suggests that enculturated apes produce more complex

\footnotetext{
${ }^{2}$ Imitation is another process that is likely to factor into ape gesturing, although studies have not found evidence that it plays a significant role in the development of gesture repertoires (Call \& Tomasello, 2007).
} 
action pantomimes than apes without such experience. One possibility is that enculturated apes are able to produce these more complex gestures because of qualitative changes that develop in their cognition. Alternatively, because of their experience interacting with humans, they might just have a lot more complex action routines to communicate about. Indeed, enculturated apes are known to learn an impressively large number of action routines from their human social partners.

\section{Conclusion}

The research described above provides evidence that apes can indeed produce a continuum of different kinds of iconic gestures, and we underscore the need for more rigorous examination of iconicity in ape gesture research. Ultimately, however, a single mechanism is unlikely to explain behavior as varied and flexible as ape gesturing. Rather each hypothesized process may contribute to a comprehensive explanation of how gesture repertoires evolve and develop and how gestures are produced in real time. Models of ape gesture will need to incorporate innate constraints on certain gestural forms, as well as constraints that arise through the interactive dyadic process of ontogenetic ritualization. Crucially, however, models must also account for the variability and creativity that are associated with the production of ape gestures. Many ape gestures appear to reflect the partial or simulated activation of instrumental actions that are contextually relevant during the moments of communication. We propose that the expansion of such an iconic process is crucial in connecting ape gesturing to the conceptually motivated gestures that are essential to human communication. Thus this creative ability of apes may lie at the evolutionary origin of human language and gesturing.

\section{References}

Arbib, M. A. (2012) How the brain got language. Oxford: OUP.

Arbib, M. A., Liebal, K., \& Pika, S. (2008). Primate vocalization, gesture, and the evolution of human language. Current Anthropology, 49, 1053-1076.

Bolinger, D. (1986). Intonation and its parts: Melody in spoken English. Palo Alto, CA: Stanford University Press.

Call, J. \& Tomasello, M. (Eds.) (2007). The gestural communication of apes and monkeys. London: Lawrence Erlbaum Associates, Publishers.

Cook, S. W. \& Tanenhaus, M. K. (2009). Embodied communication: Speakers' gestures affect listeners' actions. Cognition, 113, 98-104.

Crawford, M. P. (1937). The cooperative solving of problems by young chimpanzees. Comparative Psychology Monograph, 14, 1-88. 
Genty, E., Breuer, T., Hobaiter, C. \& Byrne, R. W. (2009). Gestural communication of the gorilla (Gorilla gorilla): Repertoire, intentionality, and possible origins. Animal Cognition, 12, 527-546.

Hayes, K. J. and Nissen, C. H. (1971). Higher mental functions of a home-raised chimpanzee. In Behavior of nonhuman primates: Modern Research Trends, A. M. Schrier and F. Stollnitz (eds), 59-115. New York: Academic Press,

Hobaiter, C. \& Byrne, R.W. (2011). The gestural repertoire of the wild chimpanzee. Animal Cognition, 14, 745-767.

Hostetter, A. B. \& Alibali, M. W. (2008). Visible embodiment: Gestures as simulated action. Psychonomic Bulletin \& Review, 15, 495-514.

Köhler, W. (1948). The mentality of apes. London: Routledge and Kegan Paul. (Original work published in 1925).

McNeill, D. (1992). Hand in mind. Chicago: University of Chicago Press.

Perlman, M., \& Gibbs, R. W. (accepted). Iconic gestures reveal the mental imagery of a human-fostered gorilla. Journal of Mental Imagery.

Perlman, M., Tanner, J. E., \& King, B. J. (2012). A mother gorilla's flexible use of touch to direct her infant reveals continuity between gesture and action. In S. Pika \& K. Liebal (Eds.) Developments in Primate Gesture Research (pp. 55-72). John Benjamins Publishing Company.

Pika, S. and Mitani, J. C. (2006). Referential gestural communication in wild chimpanzees (Pan troglodytes). Current Biology, 16, R191-R192.

Russon, A. and Andrews, K. (2011). Orangutan pantomime: elaborating the message. Biology Letters, 7, 627-630.

Savage-Rumbaugh, E. S., Wilkerson, B. J., \& Bakeman, R. (1977). Spontaneous gestural communication among conspecifics in the pygmy chimpanzee (Pan paniscus). In G. H. Bourne (Ed.) Progress in ape research (pp. 97-116). New York: Academic Press Inc.

Savage-Rumbaugh, S., McDonald, K., Sevcik, R. A., Hopkins, W. D., \& Rupert, E. (1986). Spontaneous symbol acquisition and communicative use by pygmy chimpanzees (Pan paniscus). Journal of Experimental Psychology: General, 115, 211-235.

Tanner, J. E. \& Byrne, R. W. (1996). Representation of action through iconic gesture in a captive lowland gorilla. Current Anthropology, 37, 162-173.

Tanner, J. E., Patterson, F. G. \& Byrne, R. W. (2006). The development of spontaneous gestures in zoo-living gorillas and sign-taught gorillas: from action and location to object representation. Journal of Developmental Processes, 1, 69-102.

Tomasello, M. (2008). Origins of human communication. Cambridge: MIT Press. 